\title{
Infrared Spectra of Polychlorobenzenes
}

\author{
Earle K. Plyler, Harry C. Allen, Jr., and E. D. Tidwell
}

\begin{abstract}
The infrared spectra of 12 chlorinated benzenes have been measured from 2 to 38 microns. In addition, the spectra of benzene, monofluorobenzene, monobromobenzene, and monoiodobenzene have been included. An assignment of the fundamental bands of the monosubstituted benzenes has been made. Some of the frequencies of the other compounds have also been assigned.

The spectrum of each compound is shown in the figures, and a table containing all the observed bands is included.
\end{abstract}

\section{Introduction}

There is now available the complete group of substituted chlorobenzenes, from monochlorobenzene to hexachlorobenzene. These have been purified by the Hooker Electrochemical Co. and have been made available through R. H. Kimball. A measurement of the infrared spectra of these chlorinated benzenes will serve two purposes. First, it will serve as a catalog of the spectra of these compounds, which will be useful for identification and for intercomparison with other substituted benzenes. Second, some of the typical vibrations can be traced from one substituted molecule to another, thus showing the effect of position of the chlorine atoms in the molecules on the vibrational frequencies.

It would be a gigantic task to try to assign all the observed bands for 12 chlorinated benzenes. This has not been undertaken, but a study of some of the types of vibrations has been made and the classifications listed.

In addition to the spectra of the chlorobenzenes, there have been included the spectra of monofluorobenzene, monobromobenzene, and monoiodobenzene. These vibrational bands of monosubstituted benzenes have been classified and the wave numbers compared with those of monofluorobenzene.

\section{Experimental Methods and Results}

The spectra were measured on a prism spectrometer. Prisms of $\mathrm{NaCl}, \mathrm{KBr}$, and $\mathrm{KRS}-5$ were used for the measurements from 2 to $38 \mu$. Cell thicknesses from 0.05 to $1.6 \mathrm{~mm}$ were used in different parts of the spectrum. The cell thickness was varied in regions of strong absorption so that the position of band center could be adequately determined. Where a thinner cell was used, the results are placed on the graphs as an insert curve. Several of the chlorinated benzenes are normally solids and have been measured in solution. Carbon tetrachloride was used for the solvent from 2 to $8 \mu$, carbon disulfide from 8 to $22 \mu$, and benzene or methylcyclohexane from 22 to $38 \mu$. In some regions the absorption of several of the liquids was so intense that the spectrum was determined in solution. The bands measured under these conditions are designated on the figures. Carbon tetrachloride has an absorption band of medium intensity in the region of $6.4 \mu$, and bands of the solutes in this region could be determined only approximately. The absorption spectrum of the compounds in the $\mathrm{CCl}_{4}$ solution show the $\mathrm{CCl}_{4}$ band at $6.4 \mu$ plus any absorption of the solute.

The observed spectra of the chlorinated benzenes are shown in nine figures. The absorption spectra of benzene, fluorobenzene, chlorobenzene, bromobenzene, and iodobenzene are shown from 2 to $38 \mu$ in figures 1 and 2 . Figures 3 and 4 include the spectra of $o$-dichlorobenzene, $m$-dichlorobenzene, and $p$-dichlorobenzene. The $p$-dichlorobenzene was measured in solution. The spectra of the three trichlorobenzenes are shown in figures 5 and 6 , and the spectra of the tetrachlorobenzenes are included in figures 8 and 9 . The spectra of pentachlorobenzene and hexachlorobenzene from 2 to $15 \mu$ are shown in figure 7 and from 15 to $38 \mu$ in figure 9 .

In the region from 23 to $38 \mu$ the spectrum of hexachlorobenzene is represented by a broken line. In this case, and other spectral regions of the compounds, the broken line indicates that the spectrum is less accurately determined than for the regions of the solid line. The uncertainty arises either from atmospheric absorption or some absorption present in the solvent. Bands of low intensity in the regions of solvent absorption are probably not observed.

Table 1 is a list of the observed bands of all the compounds. The wavelength, wave number, and intensity are listed for the bands of each substance. Bands of low intensity are not included. The intensity is given in terms of percentage of transmission, and is included primarily for identification purposes. No correction has been applied for radiation loss at the cell windows, nor has the percentage of transmission been reduced to a uniform cell thickness, but sufficient data are given so that a quantitative comparison of intensities can be made. It is estimated that the wavelengths are correct to \pm 0.03 $\mu$ in the region from 2 to $15 \mu$ and to $\pm 0.06 \mu$ from 15 to $38 \mu$. 


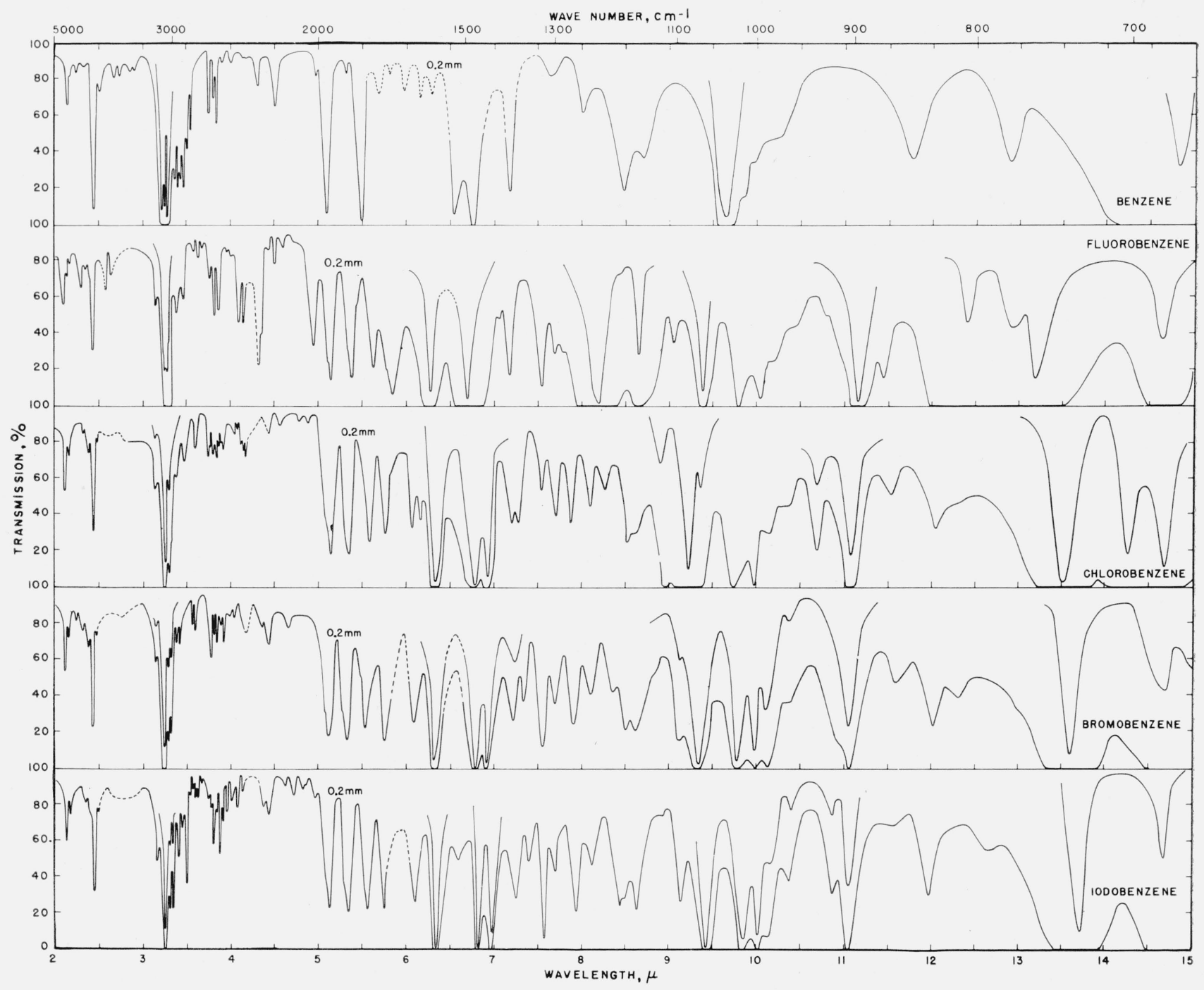

FIGURE 1. Infrared absorption spectr a of benzene, fluorobenzene, chlorobenzene, bromobenzene, and iodobenzene from 2 to 15 microns. Dotted sections represent regions of atmospheric interference.

\section{Discussion of Results}

Any complete assignment of the vibrational frequencies as determined from liquids or solutions is very difficult because the distinctive band shapes arising from the rotational fine structure are washed out. In the present case the difficulty is aggravated because the spectra beyond $38 \mu$ were not obtained. Thus many of the important low-lying fundamentals were not observed. However, with the help of the excellent work of Randle and Whiffen, ${ }^{1}$ some of the bands have been assigned. Some help was also obtained from the calculations of Garg. ${ }^{2}$

1 R. R. Randle and D. H. Whiffen, Molecular Spectroscopy Rept. of Conf.
Hydrocarbon Research Group. Inst. Petroleum

Hydrocarbon Research Group, Inst. Petroleum (Oct. 1954).
:S. N. Garg, Current Sci. 22, 298 (1953).

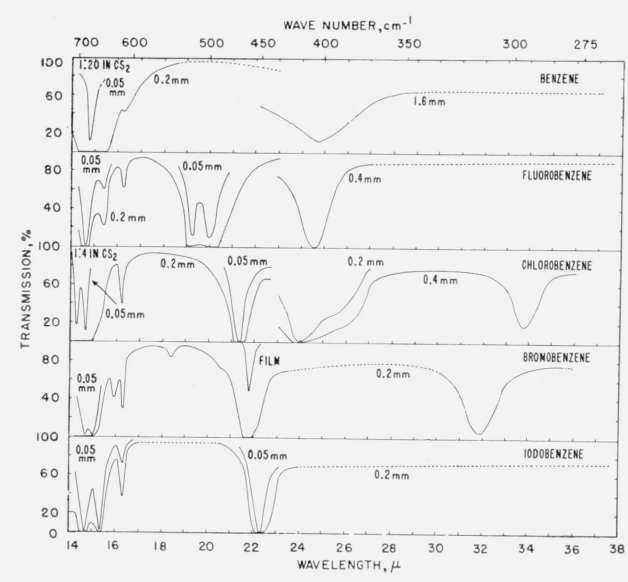

Figure 2. Continuation of the spectra of the compounds of figure 1 in the region from 14 to 38 microns. 


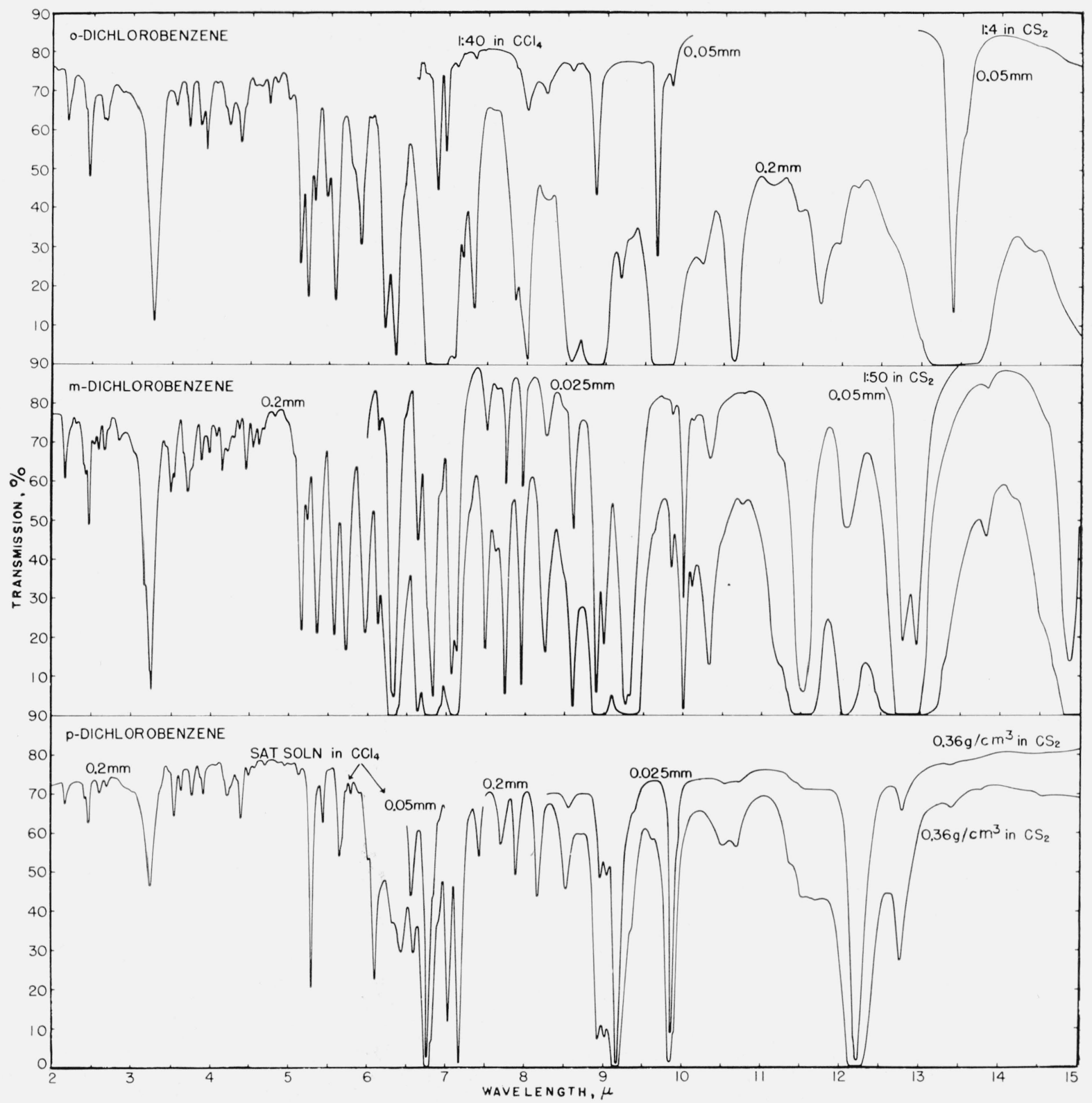

FIGURE 3. Infrared spectra of orthodichlorobenzene, metadichlorobenzene, and paradichlorobenzene from 2 to 15 microns.

The insert curves are for thinner cells or solutions in $\mathrm{CCl}_{4}$ or $\mathrm{CS}_{2}$.

FIGURE 4. Continuation of the spectra of figure 3 in the region from 14 to 38 microns.

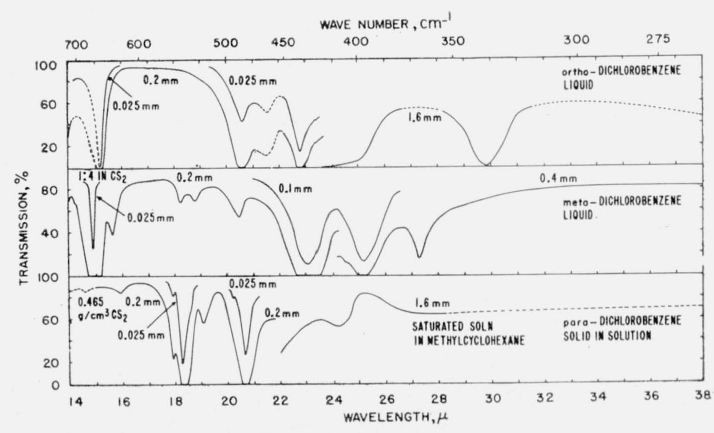




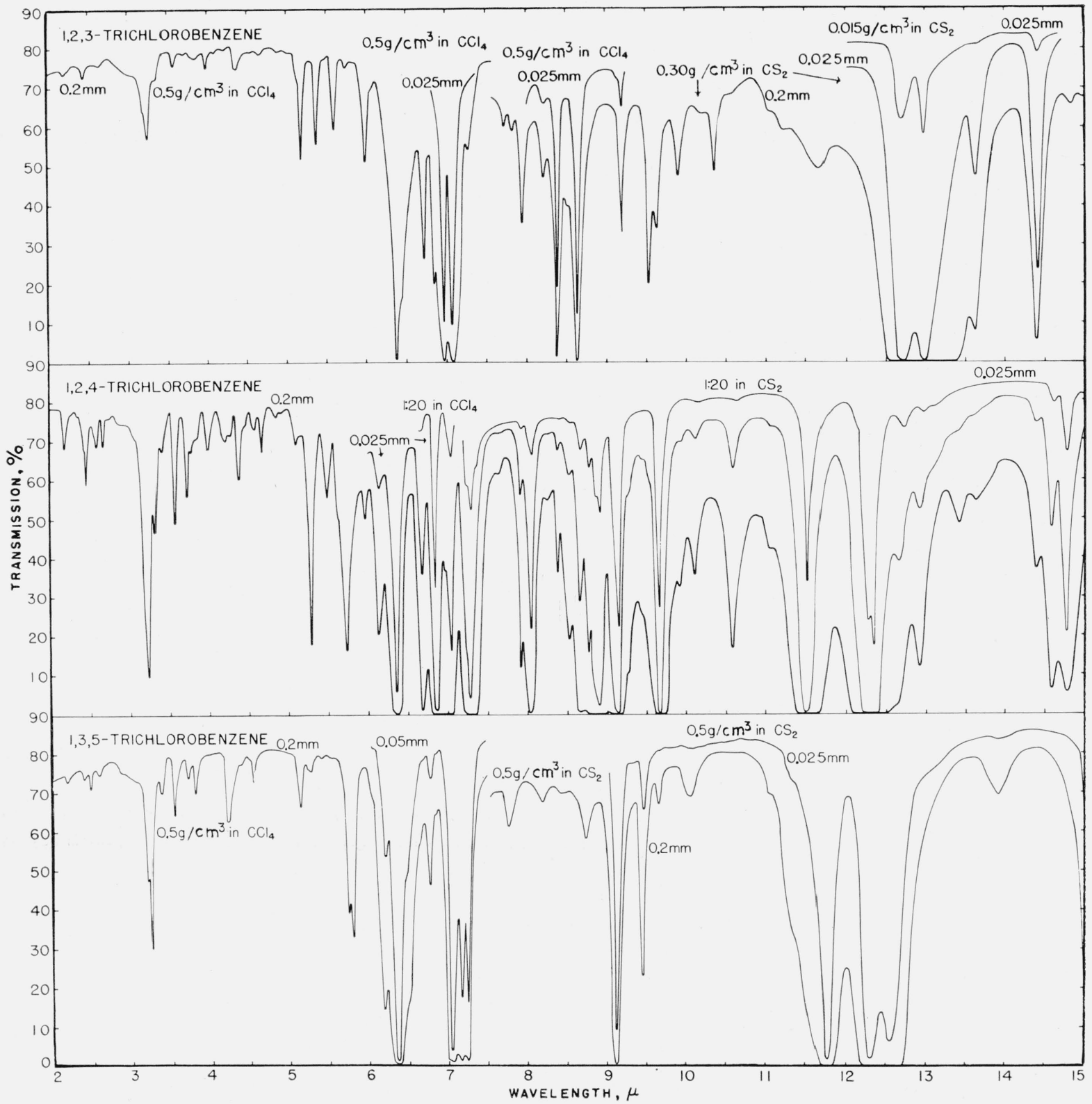

FigURE 5. Infrared spectra of 1,2,3-trichlorobenzene, 1,2,4-trichlorobenzene, and 1,3,5-trichlorobenzene from 2 to 15 microns.

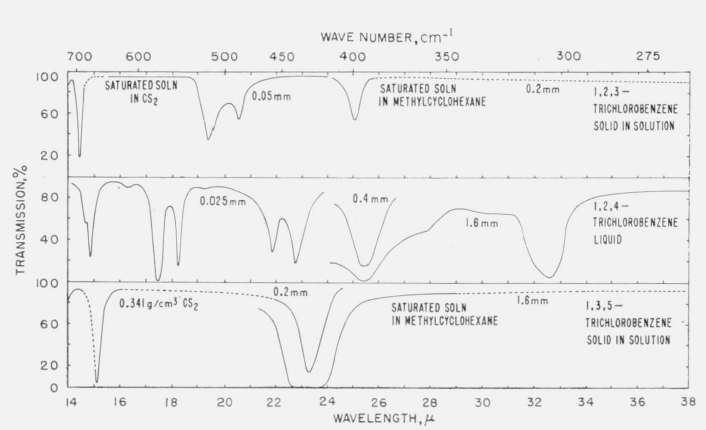

Figure 6. Continuation of the spectra of the trichlorobenzenes from 14 to 38 microns.

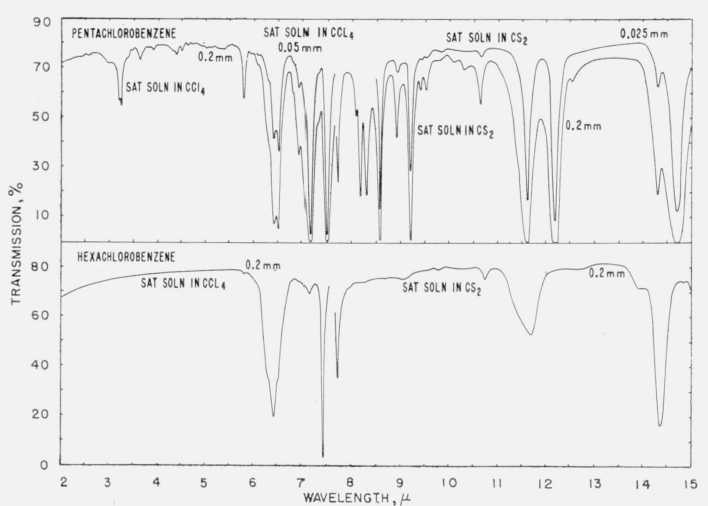

FiguRE 7. Infrared spectra of pentachlorobenzene and hexachlorobenzene from 2 to 15 microns. 


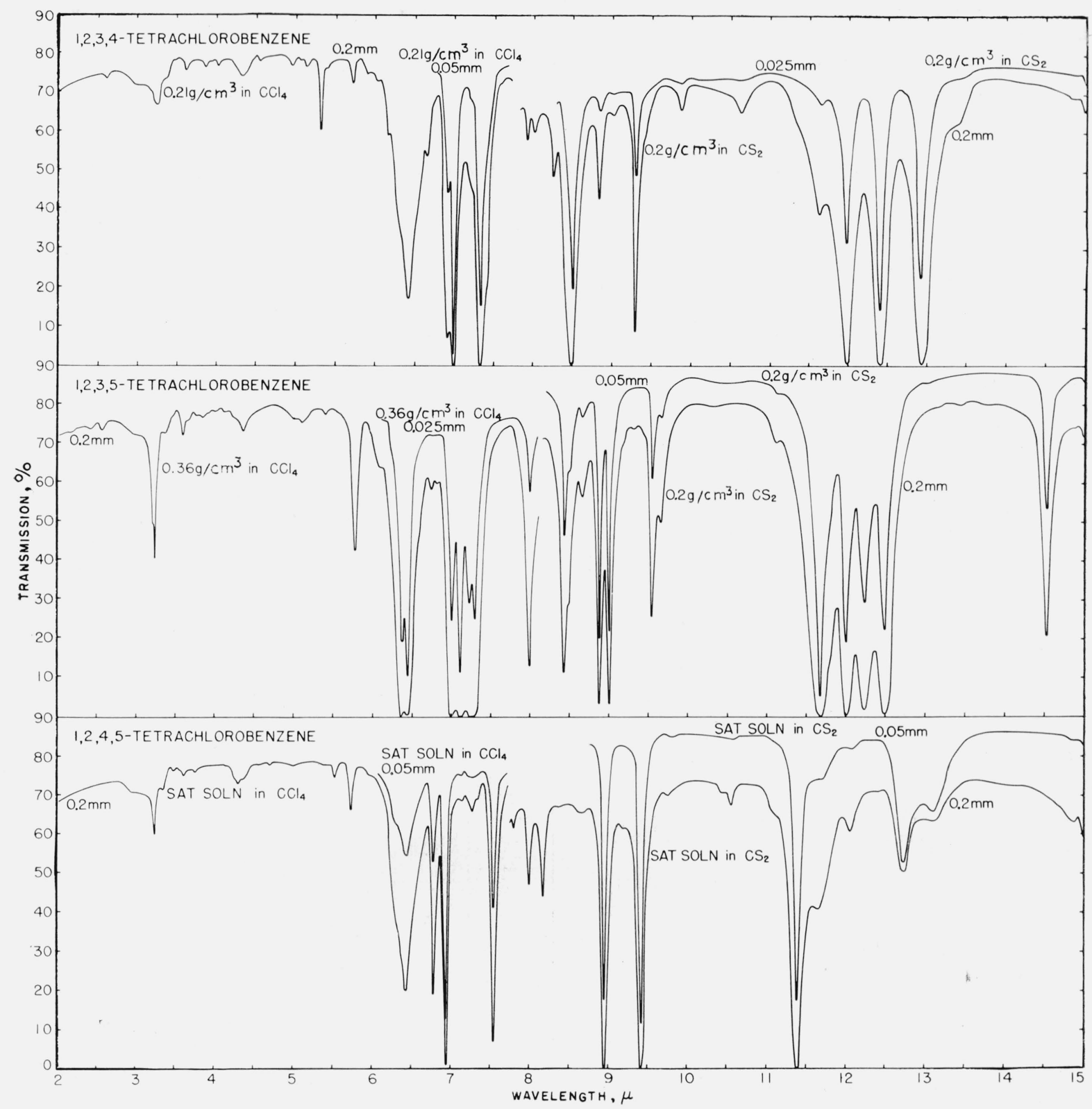

FiguRE 8. Spectra of 1,2,3,4-tetrachlorobenzene, 1,2,3,5-tetrachlorobenzene, and 1,2,4,5-tetrachlorobenzene from 2 to 15 microns.

Figire 9. Infrared spectra of the tetachlorobenzenes, pentachlorobenzene, and hexachlorobenzene from 14 to 38 mi.rons.

The dotted lines indicate that no bands of medium or strong intensity are present in that part of the spectrum.

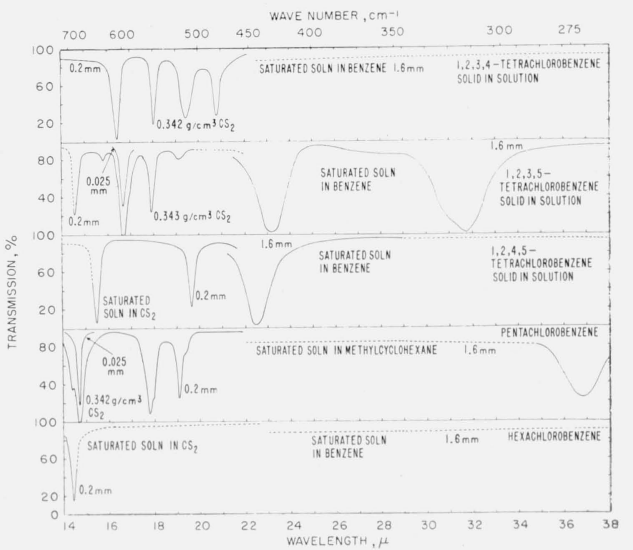


TABLE 1. Observed bands

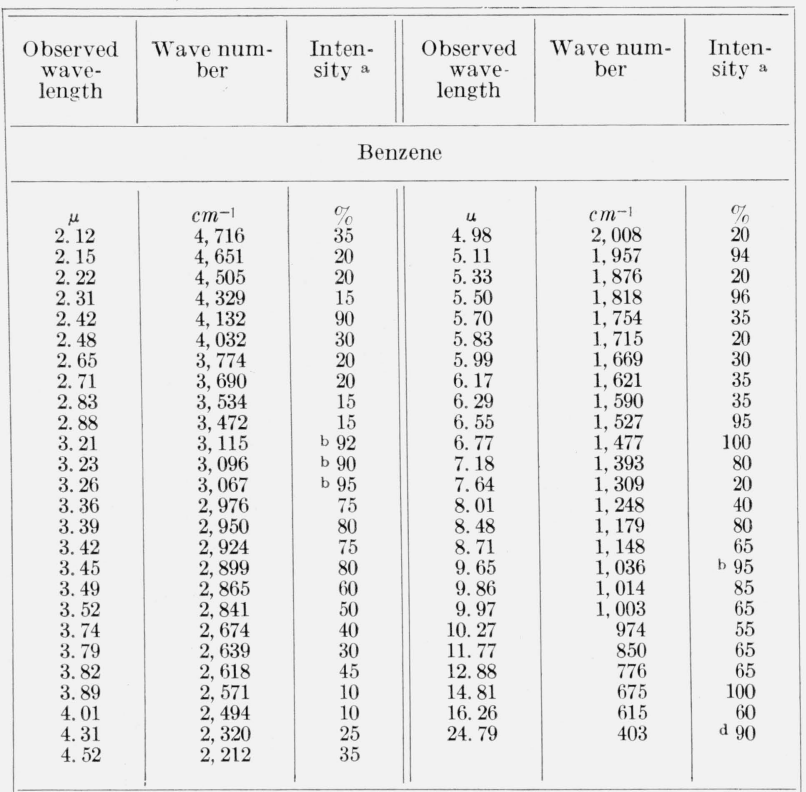

\begin{tabular}{|c|c|c|c|c|c|}
\hline \multicolumn{6}{|c|}{ Fluorobenzene } \\
\hline & 4. 808 & & 5,39 & 1855 & \\
\hline 2.00 & $\begin{array}{l}4,800 \\
4.762\end{array}$ & 30 & 5.44 & 1,838 & 45 \\
\hline 2.15 & 4,651 & 20 & 5. 63 & 1,776 & 80 \\
\hline 2. 27 & 4,405 & 35 & 5. 78 & 1,730 & 80 \\
\hline 2. 32 & $\begin{array}{l}\text { t, } \\
4,310\end{array}$ & 25 & 5.85 & $\begin{array}{l}1,709 \\
-109\end{array}$ & 90 \\
\hline 2.40 & $\begin{array}{l}\text {, o10 } \\
4,167\end{array}$ & 70 & $\begin{array}{l}6.28 \\
6.28\end{array}$ & 1,592 & $\begin{array}{l}30 \\
\text { b } 90\end{array}$ \\
\hline 2.56 & 3,906 & 35 & 6.69 & 1,495 & b 95 \\
\hline 2.62 & 3,817 & 30 & 7.07 & 1,414 & 50 \\
\hline 3. 14 & 3,185 & 45 & 7.18 & 1,393 & 80 \\
\hline 3. 22 & 3,106 & b 75 & 7.54 & 1,326 & 90 \\
\hline 3. 24 & 3,086 & b 80 & 7.70 & 1,299 & 70 \\
\hline 3. 26 & 3,067 & b 80 & 7. 79 & 1. 284 & 70 \\
\hline 3. 27 & 3,058 & b 70 & 8.15 & 1. 227 & ь 95 \\
\hline 3. 37 & 2,967 & 50 & $\begin{array}{l}8.100 \\
8.20\end{array}$ & 1,220 & b 100 \\
\hline 3. 45 & 2,899 & 45 & 8.66 & 1,155 & b 70 \\
\hline $\begin{array}{l}3.45 \\
\text { 3. } 55\end{array}$ & 2,817 & 15 & 9.07 & 1,103 & 65 \\
\hline $\begin{array}{l}3.50 \\
\text { 3. } 57\end{array}$ & 2,801 & 15 & $\begin{array}{l}9.04 \\
9.40\end{array}$ & $\begin{array}{l}1,105 \\
1,054\end{array}$ & $\begin{array}{c}50 \\
\text { b } 90\end{array}$ \\
\hline $\begin{array}{l}3.62 \\
3.62\end{array}$ & $\begin{array}{l}2,801 \\
2,762\end{array}$ & $\begin{array}{l}10 \\
20\end{array}$ & $\begin{array}{l}9.40 \\
9.81\end{array}$ & $\begin{array}{l}1,004 \\
1,019\end{array}$ & 100 \\
\hline $\begin{array}{l}5.02 \\
3.66\end{array}$ & 2,732 & 15 & $\begin{array}{r}9.81 \\
10.06\end{array}$ & $\begin{array}{r}1,019 \\
994\end{array}$ & 80 \\
\hline 3. 75 & 2,667 & 30 & 10.11 & $\begin{array}{l}837 \\
989\end{array}$ & 75 \\
\hline 3. 80 & 2,632 & 50 & & & \\
\hline 3. 85 & 2,597 & 45 & & & \\
\hline 3. 95 & 2,532 & 15 & & & \\
\hline 4. 60 & 2,500 & 15 & 11. 16 & 896 & b 95 \\
\hline 4. 09 & 2,445 & 55 & 11.46 & 873 & 80 \\
\hline 4. 11 & 2,433 & 55 & 12. 39 & 807 & b 55 \\
\hline 4. 16 & 2,404 & 55 & 12.45 & 803 & b 40 \\
\hline 4. 33 & 2,309 & 80 & 12.93 & 773 & b 55 \\
\hline 4.35 & 2,299 & 60 & 13.18 & 759 & b 85 \\
\hline 4. 44 & 2. 252 & 15 & 14. 64 & 683 & b 95 \\
\hline 4. 52 & 2,212 & 25 & 15. 53 & 644 & b 40 \\
\hline $\begin{array}{l}\text { 4. } 61 \\
\text { 4. }\end{array}$ & 2,169 & 15 & 16. 28 & 614 & 40 \\
\hline $\begin{array}{l}\text { t. } \\
\text { 4. } 95 \\
\text { a }\end{array}$ & 2,020 & 65 & 19. 26 & 519 & $\begin{array}{l}\text { b } 90 \\
\text {. }\end{array}$ \\
\hline $\begin{array}{l}\text { 5. } 11 \\
\text { 5. }\end{array}$ & $\begin{array}{l}1,957 \\
\end{array}$ & 75 & 20.00 & 500 & b 90 \\
\hline 5.15 & 1,942 & 85 & 24.56 & 407 & e 100 \\
\hline 5. 34 & 1,873 & 70 & & & \\
\hline
\end{tabular}

\begin{tabular}{|l|l|l|l|l|l|}
\hline \multicolumn{7}{|c|}{ Chlorobenzene } \\
\hline & \multicolumn{7}{|c|}{} \\
\hline 2.08 & 4,808 & 50 & 4.11 & 2,433 & 25 \\
2.13 & 4,695 & 30 & 4.14 & 2,416 & 30 \\
2.28 & 4,386 & 15 & 4.17 & 2.398 & 30 \\
2.35 & 4,255 & 25 & 4.44 & 2,252 & 20 \\
2.41 & 4,149 & 70 & 4.56 & 2,193 & 15 \\
2.45 & 4,082 & 20 & 4.78 & 2,092 & 10 \\
3.12 & 3,205 & 45 & 4.89 & 2,045 & 10 \\
3.24 & 3,086 & 100 & 5.14 & 1,946 & 80 \\
3.28 & 3,049 & 95 & 5.35 & 1,869 & 80 \\
3.36 & 2,976 & 40 & 5.59 & 1,789 & 75 \\
3.45 & 2,899 & 30 & 5.76 & 1,736 & 70 \\
3.58 & 2,793 & 25 & 6.07 & 1,647 & 70 \\
3.72 & 2,68 & 30 & 6.16 & 1,623 & 65 \\
3.75 & 2,667 & 25 & 6.33 & 1,580 & b 95 \\
3.78 & 2,646 & 30 & 6.78 & 1,475 & b 95 \\
3.82 & 2,618 & 30 & 6.92 & 1,445 & b 90 \\
3.85 & 2,597 & 25 & 7.20 & 1,389 & 60 \\
3.87 & 2,584 & 25 & 7.27 & 1,376 & 60 \\
3.90 & 2,564 & 25 & 7.54 & 1,326 & 45 \\
4.05 & 2,469 & 15 & 7.70 & 1,299 & 60 \\
4.08 & 2,451 & 15 & 7.87 & 1,271 & 65 \\
\hline
\end{tabular}

See footnotes at end of table.
Table 1. Observed bands-Continued

\begin{tabular}{|c|c|c||c|c|c|}
\hline $\begin{array}{c}\text { Observed } \\
\text { wave- } \\
\text { length }\end{array}$ & $\begin{array}{c}\text { Wave num- } \\
\text { ber }\end{array}$ & $\begin{array}{c}\text { Inten- } \\
\text { sity a }\end{array}$ & $\begin{array}{c}\text { Observed } \\
\text { wave- } \\
\text { length }\end{array}$ & $\begin{array}{c}\text { Wave num- } \\
\text { ber }\end{array}$ & $\begin{array}{c}\text { Inten- } \\
\text { sity a }\end{array}$ \\
\hline
\end{tabular}

Chlorobenzene-Continue d

\begin{tabular}{|c|c|r|r|r|r|}
\hline$\mu$ & $c m^{-1}$ & $\%$ & $\mu$ & $c m^{-1}$ & $\%$ \\
8.10 & 1,235 & 55 & 11.08 & 903 & b 80 \\
8.26 & 1,211 & 45 & 11.54 & 867 & 50 \\
8.51 & 1,175 & 75 & 12.05 & 830 & 65 \\
8.90 & 1,124 & 100 & 13.52 & 740 & b 95 \\
9.22 & 1,085 & b 90 & 14.25 & 702 & b 85 \\
9.36 & 1,068 & b 40 & 14.66 & 682 & b 90 \\
9.74 & 1,027 & 100 & 16.22 & 617 & 60 \\
9.98 & 1,002 & 100 & 21.32 & 469 & b 100 \\
10.16 & 984 & 65 & 23.94 & 418 & 100 \\
\hline 10.69 & 935 & 80 & 33.78 & 393 & 70 \\
& & & & 296 & 80
\end{tabular}

\begin{tabular}{|c|c|c|c|c|c|}
\hline \multicolumn{6}{|c|}{ Bromobenzene } \\
\hline $\begin{array}{l}2.09 \\
2.13 \\
2.21 \\
2.29 \\
2.35 \\
2.40 \\
2.44 \\
2.73 \\
3.13 \\
3.22 \\
3.27 \\
3.31 \\
3.36 \\
3.40 \\
3.54 \\
3.57 \\
3.76 \\
3.79 \\
3.82 \\
3.87 \\
3.90 \\
3.97 \\
4.04 \\
4.18 \\
4.36 \\
4.44 \\
4.67 \\
5.08 \\
5.12 \\
5.28 \\
5.33 \\
5.48 \\
5.54 \\
5.76\end{array}$ & $\begin{array}{l}4,785 \\
4,695 \\
4,525 \\
4,367 \\
4,255 \\
4,167 \\
4,098 \\
3,663 \\
3,195 \\
3,106 \\
3,058 \\
3,021 \\
2,976 \\
2,941 \\
2,825 \\
2,801 \\
2,660 \\
2,639 \\
2,618 \\
2,584 \\
2,564 \\
2,519 \\
2,475 \\
2,392 \\
2,293 \\
2,252 \\
2,141 \\
1,969 \\
1,953 \\
1,894 \\
1,876 \\
1,824 \\
1,805 \\
1,736\end{array}$ & $\begin{array}{r}50 \\
30 \\
20 \\
25 \\
35 \\
80 \\
25 \\
15 \\
45 \\
100 \\
85 \\
80 \\
30 \\
30 \\
20 \\
25 \\
40 \\
30 \\
35 \\
20 \\
35 \\
15 \\
15 \\
25 \\
25 \\
35 \\
25 \\
70 \\
80 \\
70 \\
80 \\
50 \\
75 \\
85\end{array}$ & $\begin{array}{r}6.09 \\
6.30 \\
6.78 \\
6.91 \\
7.21 \\
7.33 \\
7.55 \\
7.69 \\
7.91 \\
8.10 \\
8.36 \\
8.50 \\
8.62 \\
9.12 \\
9.34 \\
9.78 \\
9.98 \\
10.11 \\
10.37 \\
11.05 \\
11.59 \\
12.02 \\
12.31 \\
13.60 \\
14.68 \\
15.00 \\
15.94 \\
16.30 \\
18.36 \\
20.60 \\
21.81 \\
22.15 \\
31.87\end{array}$ & $\begin{array}{r}1,642 \\
1,585 \\
1,475 \\
1,447 \\
1,387 \\
1,364 \\
1,324 \\
1,300 \\
1,264 \\
1,235 \\
1,196 \\
1,176 \\
1,160 \\
1,097 \\
1,071 \\
1,023 \\
1,002 \\
989 \\
964 \\
905 \\
863 \\
832 \\
812 \\
735 \\
681 \\
667 \\
627 \\
614 \\
545 \\
485 \\
459 \\
452 \\
314\end{array}$ & $\begin{array}{r}70 \\
\text { b } 95 \\
\text { b } 100 \\
\text { b } 95 \\
75 \\
60 \\
85 \\
60 \\
75 \\
60 \\
60 \\
80 \\
80 \\
85 \\
\text { b } 95 \\
\text { b } 95 \\
\text { b } 90 \\
\text { b } 65 \\
60 \\
100 \\
50 \\
80 \\
60 \\
\text { b } 90 \\
\text { b } 95 \\
\text { b } 95 \\
60 \\
70 \\
15 \\
30 \\
100 \\
90 \\
90\end{array}$ \\
\hline \multicolumn{6}{|c|}{ Iodobenzene } \\
\hline $\begin{array}{l}2.11 \\
2.15 \\
2.32 \\
2.42 \\
2.46 \\
3.14 \\
3.23 \\
3.29 \\
3.32 \\
3.38 \\
3.42 \\
3.48 \\
3.52 \\
3.55 \\
3.57 \\
3.61 \\
3.65 \\
3.72 \\
3.75 \\
3.78 \\
3.82 \\
3.85 \\
3.89 \\
3.93 \\
4.01 \\
4.07 \\
4.14 \\
4.37 \\
4.43 \\
4.63 \\
4.72 \\
4.83 \\
4.86 \\
4.96 \\
5.13\end{array}$ & $\begin{array}{l}4,739 \\
4,651 \\
4,310 \\
4,132 \\
4,065 \\
3,185 \\
3,096 \\
3,040 \\
3,012 \\
2,959 \\
2,924 \\
2,874 \\
2,841 \\
2,817 \\
2,801 \\
2,770 \\
2,740 \\
2,688 \\
2,667 \\
2,646 \\
2,618 \\
2,597 \\
2,571 \\
2,545 \\
2,494 \\
2,457 \\
2,415 \\
2,288 \\
2,257 \\
2,160 \\
2,119 \\
2,070 \\
2,058 \\
2,016 \\
1,949\end{array}$ & $\begin{aligned} 40 \\
25 \\
20 \\
70 \\
25 \\
50 \\
\text { b } 90 \\
80 \\
80 \\
50 \\
30 \\
65 \\
15 \\
10 \\
15 \\
15 \\
10 \\
20 \\
25 \\
45 \\
39 \\
50 \\
30 \\
25 \\
15 \\
20 \\
10 \\
20 \\
25 \\
10 \\
15 \\
10 \\
10 \\
20 \\
80\end{aligned}$ & $\begin{array}{r}5.34 \\
5.56 \\
5.75 \\
6.10 \\
6.34 \\
6.60 \\
6.81 \\
6.97 \\
7.25 \\
7.40 \\
7.57 \\
7.70 \\
7.94 \\
8.12 \\
8.44 \\
8.51 \\
8.63 \\
8.95 \\
9.14 \\
9.43 \\
9.85 \\
10.03 \\
10.16 \\
10.38 \\
10.87 \\
11.06 \\
11.57 \\
11.97 \\
12.66 \\
13.71 \\
14.17 \\
14.68 \\
15.31 \\
16.32 \\
22.32\end{array}$ & $\begin{array}{r}1,873 \\
1,799 \\
1,739 \\
1,639 \\
1,577 \\
1,515 \\
1,468 \\
1,435 \\
1,379 \\
1,351 \\
1,321 \\
1,299 \\
1,259 \\
1,232 \\
1,185 \\
1,175 \\
1,159 \\
1,117 \\
1,094 \\
1,060 \\
1,015 \\
997 \\
984 \\
963 \\
920 \\
904 \\
864 \\
835 \\
790 \\
729 \\
707 \\
681 \\
653 \\
613 \\
448\end{array}$ & $\begin{array}{r}80 \\
80 \\
80 \\
75 \\
\text { b } 100 \\
45 \\
\text { b } 100 \\
\text { b } 90 \\
70 \\
50 \\
90 \\
55 \\
80 \\
50 \\
70 \\
65 \\
70 \\
25 \\
65 \\
\text { b } 100 \\
\text { b } 90 \\
\text { b } 90 \\
90 \\
55 \\
60 \\
\text { b } 55 \\
25 \\
65 \\
60 \\
\text { b } 85 \\
90 \\
\text { b } 95 \\
\text { b } 95 \\
65 \\
\text { b } 100\end{array}$ \\
\hline
\end{tabular}


TABLE 1. Observed bands-Continued

\begin{tabular}{|c|c|c|c|c|c|}
\hline $\begin{array}{l}\text { Observed } \\
\text { wave- } \\
\text { length }\end{array}$ & $\begin{array}{l}\text { Wave num- } \\
\text { ber }\end{array}$ & $\begin{array}{l}\text { Inten- } \\
\text { sity a }\end{array}$ & $\begin{array}{c}\text { Observed } \\
\text { wave- } \\
\text { length }\end{array}$ & $\begin{array}{l}\text { Wave num- } \\
\text { ber }\end{array}$ & $\begin{array}{l}\text { Inten- } \\
\text { sity } \mathrm{a}\end{array}$ \\
\hline \multicolumn{6}{|c|}{ Orthodichlorobenzene } \\
\hline $\begin{array}{c}\mu \\
2.17 \\
2.41 \\
2.44 \\
2.62 \\
2.65 \\
3.25 \\
3.53 \\
3.69 \\
3.85 \\
3.91 \\
4.17 \\
4.23 \\
\text { e } 4.38 \\
4.41 \\
4.55 \\
4.62 \\
4.73 \\
4.84 \\
4.98 \\
5.13 \\
5.24 \\
5.31 \\
5.47 \\
5.58 \\
5.79\end{array}$ & $\begin{array}{l}c m-1 \\
4,608 \\
4,149 \\
4,098 \\
3,817 \\
3,775 \\
3,077 \\
2,833 \\
2,710 \\
2,597 \\
2,558 \\
2,398 \\
2,364 \\
2,283 \\
2,268 \\
2,198 \\
2,164 \\
2,114 \\
2,066 \\
2,008 \\
1,949 \\
1,908 \\
1,883 \\
1,828 \\
1,792 \\
1,727\end{array}$ & $\begin{array}{l}\% \\
35 \\
35 \\
50 \\
35 \\
35 \\
90 \\
35 \\
40 \\
40 \\
45 \\
35 \\
40 \\
45 \\
35 \\
30 \\
30 \\
35 \\
30 \\
35 \\
75 \\
85 \\
60 \\
60 \\
85 \\
50\end{array}$ & $\begin{array}{r}\mu \\
5.90 \\
6.02 \\
6.21 \\
\mathrm{e} 6.34 \\
6.74 \\
6.86 \\
6.97 \\
7.11 \\
7.19 \\
7.33 \\
7.47 \\
\mathrm{e} 7.86 \\
\mathrm{e} 7.99 \\
8.57 \\
8.86 \\
9.64 \\
\mathrm{e} 9.82 \\
10.63 \\
13.38 \\
13.52 \\
15.18 \\
20.60 \\
21.56 \\
22.82 \\
30.85\end{array}$ & $\begin{array}{r}c m-1 \\
1,695 \\
1,661 \\
1,610 \\
1,577 \\
1,484 \\
1,458 \\
1,435 \\
1,406 \\
1,391 \\
1,364 \\
1,339 \\
1,272 \\
1,252 \\
1,167 \\
1,129 \\
1,037 \\
1,018 \\
941 \\
747 \\
739 \\
659 \\
485 \\
464 \\
438 \\
324\end{array}$ & $\begin{array}{r}\% \\
70 \\
40 \\
90 \\
95 \\
\text { b } 25 \\
\text { b } 55 \\
\text { b } 45 \\
95 \\
75 \\
75 \\
40 \\
40 \\
85 \\
100 \\
100 \\
\text { b } 55 \\
\text { b } 70 \\
\text { b } 30 \\
100 \\
\text { b } 85 \\
\text { b } 40 \\
\text { b } 100 \\
100 \\
90 \\
900 \\
100 \\
100\end{array}$ \\
\hline \multicolumn{6}{|c|}{ Metadichlorobenzene } \\
\hline $\begin{array}{r}2.15 \\
2.29 \\
2.39 \\
2.40 \\
2.45 \\
2.51 \\
2.57 \\
2.64 \\
2.83 \\
3.02 \\
3.17 \\
3.22 \\
3.24 \\
\text { e } 3.48 \\
3.51 \\
3.64 \\
3.70 \\
3.74 \\
3.86 \\
3.96 \\
4.08 \\
4.16 \\
4.22 \\
4.30 \\
4.46 \\
4.55 \\
4.62 \\
4.68 \\
4.82 \\
5.08 \\
5.17 \\
5.23 \\
5.37 \\
5.58 \\
\text { 5. } 5.73 \\
5.97 \\
5.99 \\
5.13\end{array}$ & $\begin{array}{l}4,651 \\
4,367 \\
4,184 \\
4,167 \\
4,082 \\
3,984 \\
3,891 \\
3,788 \\
3,534 \\
3,311 \\
3,155 \\
3,106 \\
3,086 \\
2,874 \\
2,849 \\
2,747 \\
2,703 \\
2,674 \\
2,591 \\
2,525 \\
2,451 \\
2,404 \\
2,370 \\
2,326 \\
2,242 \\
2,198 \\
2,164 \\
2,137 \\
2,075 \\
1,968 \\
1,934 \\
1,912 \\
1,862 \\
1,792 \\
1,745 \\
1,675 \\
1,669 \\
1,631\end{array}$ & $\begin{array}{l}40 \\
25 \\
35 \\
35 \\
50 \\
30 \\
30 \\
30 \\
25 \\
30 \\
70 \\
90 \\
95 \\
45 \\
40 \\
35 \\
45 \\
40 \\
35 \\
35 \\
30 \\
40 \\
35 \\
30 \\
30 \\
40 \\
30 \\
30 \\
25 \\
35 \\
80 \\
50 \\
80 \\
80 \\
85 \\
80 \\
75 \\
75\end{array}$ & $\begin{array}{r}\text { e } 6.33 \\
\text { e } 6.64 \\
6.82 \\
6.90 \\
7.07 \\
7.18 \\
7.49 \\
7.63 \\
7.73 \\
7.94 \\
\mathrm{f} 8.25 \\
8.48 \\
8.59 \\
8.88 \\
8.98 \\
9.23 \\
9.25 \\
9.31 \\
\mathrm{f} 9.62 \\
9.83 \\
10.00 \\
10.11 \\
10.32 \\
10.48 \\
10.74 \\
\mathrm{f} 11.20 \\
11.51 \\
12.06 \\
12.77 \\
12.92 \\
14.86 \\
15.64 \\
18.25 \\
18.79 \\
20.46 \\
23.09 \\
25.19 \\
27.29\end{array}$ & $\begin{array}{r}1,580 \\
1,506 \\
1,466 \\
1,449 \\
1,414 \\
1,393 \\
1,335 \\
1,311 \\
1,295 \\
1,259 \\
1,212 \\
1,179 \\
1,164 \\
1,126 \\
1,114 \\
1,083 \\
1,081 \\
1,074 \\
1,040 \\
1,017 \\
1,000 \\
989 \\
969 \\
954 \\
931 \\
893 \\
869 \\
829 \\
783 \\
774 \\
673 \\
639 \\
548 \\
532 \\
489 \\
433 \\
397 \\
366\end{array}$ & $\begin{array}{r}100 \\
95 \\
100 \\
95 \\
100 \\
100 \\
85 \\
60 \\
95 \\
90 \\
85 \\
60 \\
95 \\
100 \\
100 \\
100 \\
100 \\
100 \\
55 \\
60 \\
100 \\
65 \\
85 \\
55 \\
45 \\
85 \\
100 \\
100 \\
100 \\
100 \\
100 \\
60 \\
30 \\
30 \\
45 \\
100 \\
100 \\
80\end{array}$ \\
\hline \multicolumn{6}{|c|}{ Paradichlorobenzene } \\
\hline $\begin{array}{r}2.15 \\
2.40 \\
2.45 \\
2.59 \\
2.68 \\
3.22 \\
\text { e. } 32 \\
3.61 \\
3.75 \\
3.85 \\
3.90 \\
4.19\end{array}$ & $\begin{array}{l}4,651 \\
4,167 \\
4,082 \\
3,861 \\
3,731 \\
3,106 \\
2,841 \\
2,770 \\
2,667 \\
2,597 \\
2,564 \\
2,387\end{array}$ & $\begin{array}{l}35 \\
30 \\
35 \\
30 \\
30 \\
55 \\
35 \\
30 \\
30 \\
25 \\
30 \\
30\end{array}$ & $\begin{array}{r}4.22 \\
4.26 \\
4.34 \\
\text { e. } 49 \\
4.50 \\
4.57 \\
4.70 \\
4.95 \\
5.12 \\
5.28 \\
5.43 \\
5.65\end{array}$ & $\begin{array}{l}2,370 \\
2,347 \\
2,304 \\
2,27 \times \\
2,222 \\
2,188 \\
2,128 \\
2,020 \\
1,953 \\
1,894 \\
1,842 \\
1,770\end{array}$ & $\begin{array}{l}30 \\
30 \\
25 \\
35 \\
20 \\
20 \\
20 \\
20 \\
25 \\
80 \\
35 \\
45\end{array}$ \\
\hline
\end{tabular}

TABLE 1, Observed bands-Continued

\begin{tabular}{|c|c|c||c|c|c}
\hline $\begin{array}{c}\text { Observed } \\
\text { wave- } \\
\text { length }\end{array}$ & $\begin{array}{c}\text { Wave num- } \\
\text { ber }\end{array}$ & $\begin{array}{c}\text { Inten- } \\
\text { sity } \mathbf{s}\end{array}$ & $\begin{array}{c}\text { Observed } \\
\text { wave- } \\
\text { length }\end{array}$ & $\begin{array}{c}\text { Wave num- } \\
\text { ber }\end{array}$ & $\begin{array}{c}\text { Inten- } \\
\text { sity s }\end{array}$ \\
\hline
\end{tabular}

Paradichlorobenzene--Continued

\begin{tabular}{r|l|r|r|r|r}
$\mu$ & $c m^{-1}$ & $\%$ & & & \\
5.68 & 1,760 & 45 & 9.18 & $c m^{-1}$ & $\%$ \\
e 5.72 & 1,748 & 30 & 9.36 & 1,089 & 100 \\
5.90 & 1,695 & 30 & 9.85 & 1,068 & 65 \\
6.01 & 1,664 & 50 & 9.90 & 1,015 & 100 \\
6.08 & 1,645 & 80 & 10.52 & 951 & 90 \\
e 6.32 & 1,582 & 70 & 10.69 & 935 & 40 \\
6.77 & 1,477 & 70 & 12.21 & 819 & 100 \\
6.82 & 1,466 & 100 & 12.75 & 784 & 70 \\
6.89 & 1,451 & 95 & 13.40 & 746 & 25 \\
7.02 & 1,424 & 85 & 14.59 & 685 & 20 \\
7.16 & 1,397 & 100 & 15.91 & 628 & 20 \\
7.43 & 1,346 & 45 & 17.92 & 558 & 80 \\
7.77 & 1,287 & 40 & 18.28 & 547 & 100 \\
7.91 & 1,264 & 50 & 19.08 & 524 & 45 \\
8.18 & 1,222 & 55 & 20.21 & 495 & 100 \\
853 & 1,172 & 55 & 20.67 & 484 & 45 \\
8.94 & 1,118 & 90 & 24.18 & 414 & 45 \\
9.03 & 1,107 & 90 & & & \\
& & & & &
\end{tabular}

1,2,3-Trichlorobenzene

\begin{tabular}{|c|c|c|c|c|c|}
\hline 2.17 & 4,608 & 25 & 7.83 & 1,277 & 40 \\
\hline 2.42 & 4,132 & 25 & $\begin{array}{r}7.90 \\
7.90\end{array}$ & 1. 266 & 65 \\
\hline 2.61 & 3,831 & 25 & ค 8.22 & 1, 216 & 50 \\
\hline 3. 20 & 3,125 & 40 & 8. 38 & 1,193 & 100 \\
\hline 3. 24 & 3,086 & 45 & 8.52 & 1,174 & 65 \\
\hline 3.34 & 2,994 & 25 & 8.63 & 1,159 & 100 \\
\hline 3.55 & 2,817 & 20 & 9.19 & 1,088 & 65 \\
\hline 3.96 & 2,525 & 25 & 9.53 & 1,049 & 80 \\
\hline 4. 67 & 2,141 & 20 & f 9.63 & 1,038 & 65 \\
\hline 5. 18 & 1,930 & 50 & 9.91 & 1,009 & 50 \\
\hline 5. 38 & 1,859 & 45 & 10. 21 & 979 & 40 \\
\hline 5. 60 & 1,786 & 40 & 10.37 & 964 & 50 \\
\hline 5. 99 & 1,669 & 50 & f 12.70 & 787 & 100 \\
\hline 6.72 & 1,488 & 75 & 12.98 & 770 & 100 \\
\hline 6.85 & 1,460 & 80 & 13. 63 & 733 & 90 \\
\hline 6.97 & 1,435 & 100 & 14.39 & 695 & 95 \\
\hline 7.07 & 1,414 & 100 & 19.42 & 515 & 65 \\
\hline е 7.27 & 1,376 & 45 & 20.57 & 486 & 45 \\
\hline 7. 73 & 1,294 & 40 & 25.06 & 399 & 45 \\
\hline \multicolumn{6}{|c|}{ 1,2,4-Trichlorobenzene } \\
\hline 2.15 & 4,651 & 35 & 8.03 & 1,245 & 100 \\
\hline 2.40 & 4,167 & 35 & 8.37 & 1. 195 & 65 \\
\hline 2. 44 & 4,098 & 40 & 8.52 & 1,173 & 80 \\
\hline 2. 54 & 3,937 & 30 & 8.65 & 1,156 & 95 \\
\hline 2.64 & 3,788 & 30 & 8.76 & 1,142 & 100 \\
\hline 3. 21 & 3,115 & 90 & 8.86 & 1,129 & 100 \\
\hline 3.31 & 3,021 & 55 & 8. 91 & 1,122 & 100 \\
\hline 3.56 & 2,809 & 50 & f 9.14 & 1,094 & 100 \\
\hline 3.71 & 2,695 & 45 & 9.25 & 1,081 & 90 \\
\hline 3.76 & 2,660 & 35 & f 9.65 & 1,036 & 100 \\
\hline 3.82 & 2,618 & 30 & 9.90 & 1. 010 & 70 \\
\hline 3.97 & 2,519 & 35 & 10.09 & 991 & 65 \\
\hline 4. 21 & 2,375 & 30 & 10.58 & 945 & 85 \\
\hline 4. 28 & 2, 336 & 30 & 11.05 & 905 & 60 \\
\hline 4.58 & 2,183 & 40 & f 11.52 & 868 & 100 \\
\hline 4. 67 & 2,141 & 25 & 12.27 & 815 & 100 \\
\hline 4. 86 & 2,058 & 35 & 12.33 & 811 & 100 \\
\hline 5.11 & 1,957 & 30 & 12.65 & 790 & 95 \\
\hline 5.29 & 1,890 & 85 & 12.92 & 774 & 85 \\
\hline 5.50 & 1,818 & 45 & 13.41 & 746 & 50 \\
\hline 5.65 & 1,770 & 50 & 13. 62 & 734 & 45 \\
\hline e 5.73 & 1,745 & 85 & 14.39 & 695 & 65 \\
\hline 5.97 & 1,675 & 50 & 14.58 & 686 & 95 \\
\hline 6.14 & 1,629 & 80 & 14.77 & 677 & 95 \\
\hline e 6.37 & 1,570 & 100 & 16.30 & 613 & 40 \\
\hline 6.68 & 1,497 & 95 & 17.40 & 575 & 100 \\
\hline 6.84 & 1,462 & 100 & 18.16 & 551 & 100 \\
\hline 7.04 & 1,420 & 100 & 19.20 & 521 & 40 \\
\hline 7.23 & 1,383 & 100 & 21.80 & 459 & 100 \\
\hline 7. 28 & 1,374 & 100 & 22.76 & 439 & 100 \\
\hline 7.35 & 1,360 & 100 & 25.44 & 393 & d 95 \\
\hline 7.63 & 1,311 & 40 & 32.57 & 307 & d 90 \\
\hline 7. 91 & 1,264 & 85 & & & \\
\hline
\end{tabular}

See footnotes at end of table. 
TaBLE 1. Observed bands-Continued

\begin{tabular}{|c|c|c|c|c|c|}
\hline $\begin{array}{l}\text { Observed } \\
\text { wave- } \\
\text { length }\end{array}$ & $\begin{array}{c}\text { Wave num- } \\
\text { ber }\end{array}$ & $\begin{array}{l}\text { Inten- } \\
\text { sity a }\end{array}$ & $\begin{array}{l}\text { Observed } \\
\text { wave- } \\
\text { length }\end{array}$ & $\begin{array}{c}\text { Wave num- } \\
\text { ber }\end{array}$ & $\begin{array}{l}\text { Inten- } \\
\text { sity a }\end{array}$ \\
\hline \multicolumn{6}{|c|}{ 1,3,5-Trichlorobenzene } \\
\hline $\begin{array}{l}\mu \\
2.16 \\
2.38 \\
2.45 \\
2.56 \\
3.19 \\
3.23 \\
3.52 \\
3.70 \\
3.79 \\
4.23 \\
4.55 \\
5.13 \\
5.27 \\
5.42 \\
\text { e } 5.74 \\
\text { e } 5.79 \\
\text { e } 6.21 \\
\text { e } 6.37 \\
\text { e } 6.46\end{array}$ & $\begin{array}{l}c m^{-1} \\
4,630 \\
4,202 \\
4,082 \\
3,906 \\
3,135 \\
3,096 \\
2,841 \\
2,703 \\
2,639 \\
2,364 \\
2,198 \\
1,949 \\
1,898 \\
1,845 \\
1,742 \\
1,727 \\
1,610 \\
1,570 \\
1,548\end{array}$ & $\begin{array}{r}\% \\
25 \\
25 \\
25 \\
25 \\
55 \\
70 \\
35 \\
25 \\
30 \\
40 \\
30 \\
40 \\
25 \\
20 \\
60 \\
65 \\
85 \\
\text { b } 100 \\
85\end{array}$ & $\begin{array}{r}\mu \\
6.77 \\
7.04 \\
7.16 \\
7.24 \\
7.77 \\
8.19 \\
8.43 \\
8.74 \\
9.13 \\
9.46 \\
\text { f9.66 } \\
10.05 \\
11.05 \\
\text { f } 11.77 \\
12.31 \\
12.55 \\
13.93 \\
15.10 \\
23.29\end{array}$ & $\begin{array}{l}c m^{-1} \\
1,477 \\
1,420 \\
1,397 \\
1,381 \\
1,287 \\
1,221 \\
1,180 \\
1,144 \\
1,095 \\
1,057 \\
1,035 \\
995 \\
905 \\
850 \\
812 \\
797 \\
718 \\
662 \\
429\end{array}$ & $\begin{array}{r}\% \\
55 \\
\text { b } 95 \\
\text { b } 80 \\
\text { b } 80 \\
35 \\
30 \\
30 \\
40 \\
100 \\
80 \\
35 \\
30 \\
30 \\
100 \\
100 \\
100 \\
30 \\
95 \\
80\end{array}$ \\
\hline \multicolumn{6}{|c|}{ 1,2,3,4-Tetrachlorobenzene } \\
\hline $\begin{array}{r}2.59 \\
3.23 \\
3.61 \\
3.86 \\
4.03 \\
\text { e } 4.33 \\
\text { e } 4.56 \\
\text { e } 4.97 \\
5.15 \\
5.33 \\
5.42 \\
\text { e } 5.73 \\
5.92 \\
6.05 \\
6.17 \\
6.66 \\
6.93 \\
6.99 \\
7.33\end{array}$ & $\begin{array}{l}3,861 \\
3,096 \\
2,770 \\
2,591 \\
2,481 \\
2,309 \\
2,193 \\
2,012 \\
1,942 \\
1,876 \\
1,845 \\
1,745 \\
1,689 \\
1,653 \\
1,620 \\
1,502 \\
1,443 \\
1,430 \\
1,364\end{array}$ & $\begin{array}{r}25 \\
35 \\
25 \\
25 \\
25 \\
25 \\
25 \\
25 \\
25 \\
40 \\
25 \\
30 \\
25 \\
30 \\
40 \\
45 \\
95 \\
160 \\
100\end{array}$ & $\begin{array}{r}7.93 \\
8.03 \\
8.27 \\
8.52 \\
8.84 \\
9.03 \\
9.31 \\
9.40 \\
9.88 \\
10.64 \\
\mathrm{f} 11.98 \\
12.42 \\
12.92 \\
13.39 \\
16.44 \\
18.05 \\
19.43 \\
20.80\end{array}$ & $\begin{array}{r}1,261 \\
1,245 \\
1,209 \\
1,174 \\
1,131 \\
1,107 \\
1,074 \\
1,064 \\
1,012 \\
940 \\
835 \\
805 \\
774 \\
746 \\
608 \\
554 \\
515 \\
481\end{array}$ & $\begin{array}{r}40 \\
40 \\
50 \\
100 \\
55 \\
35 \\
90 \\
40 \\
35 \\
35 \\
100 \\
100 \\
100 \\
40 \\
95 \\
80 \\
70 \\
70\end{array}$ \\
\hline \multicolumn{6}{|c|}{ 1,2,3,5-Tetrachlorobenzene } \\
\hline $\begin{array}{r}2.40 \\
2.54 \\
3.20 \\
3.22 \\
\text { e } 3.35 \\
3.58 \\
3.63 \\
5.41 \\
\text { e } 5.77 \\
6.10 \\
\text { e } 6.37 \\
\text { e } 6.44 \\
6.74 \\
7.00 \\
7.10 \\
7.22 \\
7.29 \\
8.00\end{array}$ & $\begin{array}{l}4,167 \\
3,937 \\
3,125 \\
3,106 \\
2,985 \\
2,793 \\
2,755 \\
1,848 \\
1,734 \\
1,639 \\
1,570 \\
1,553 \\
1,484 \\
1,428 \\
1,408 \\
1,385 \\
1,372 \\
1,250\end{array}$ & $\begin{array}{r}25 \\
25 \\
50 \\
60 \\
25 \\
25 \\
25 \\
25 \\
55 \\
35 \\
100 \\
100 \\
45 \\
100 \\
100 \\
100 \\
100 \\
90\end{array}$ & $\begin{array}{r}8.42 \\
8.48 \\
8.66 \\
8.87 \\
8.99 \\
\text { f } 9.53 \\
9.65 \\
\text { f } 11.67 \\
\text { f } 12.00 \\
12.23 \\
12.48 \\
14.54 \\
15.79 \\
16.70 \\
17.93 \\
19.10 \\
23.10 \\
31.74\end{array}$ & $\begin{array}{l}1,188 \\
1,179 \\
1,155 \\
1,127 \\
1,112 \\
1,049 \\
1,036 \\
857 \\
833 \\
818 \\
801 \\
688 \\
633 \\
599 \\
558 \\
523 \\
431 \\
315\end{array}$ & $\begin{array}{r}£ 0 \\
70 \\
45 \\
95 \\
95 \\
75 \\
50 \\
100 \\
100 \\
160 \\
100 \\
80 \\
20 \\
100 \\
75 \\
20 \\
\mathrm{~d} 95 \\
\mathrm{~d} 95\end{array}$ \\
\hline \multicolumn{6}{|c|}{$1,2,4,5$-Tetrachlorobenzene } \\
\hline $\begin{array}{r}3.23 \\
\text { e } 4.32 \\
5.54 \\
\text { e } 5.74 \\
6.79 \\
6.95 \\
7.54 \\
7.81 \\
8.00 \\
8.13 \\
8.97\end{array}$ & $\begin{array}{l}3,096 \\
2,288 \\
1,805 \\
1,742 \\
1,473 \\
1,439 \\
1,326 \\
1,280 \\
1,250 \\
1,230 \\
1,115\end{array}$ & $\begin{array}{r}40 \\
25 \\
25 \\
35 \\
80 \\
100 \\
90 \\
40 \\
55 \\
55 \\
100\end{array}$ & $\begin{array}{r}9.42 \\
10.45 \\
10.56 \\
\text { f } 11.40 \\
12.08 \\
\text { f } 12.74 \\
13.13 \\
15.49 \\
19.63 \\
22.52\end{array}$ & $\begin{array}{r}1,062 \\
957 \\
947 \\
877 \\
828 \\
785 \\
762 \\
646 \\
509 \\
444\end{array}$ & $\begin{array}{r}100 \\
30 \\
35 \\
100 \\
40 \\
50 \\
35 \\
95 \\
80 \\
\text { d } 100\end{array}$ \\
\hline
\end{tabular}

TABLE 1. Observed bands-Continued

\begin{tabular}{|c|c|c|c|c|c|}
\hline $\begin{array}{c}\text { Observed } \\
\text { wave- } \\
\text { length }\end{array}$ & $\begin{array}{c}\text { Wave num- } \\
\text { ber }\end{array}$ & $\begin{array}{l}\text { Inten- } \\
\text { sity a }\end{array}$ & $\begin{array}{l}\text { Observed } \\
\text { wave- } \\
\text { length }\end{array}$ & $\begin{array}{l}\text { Wave num- } \\
\text { ber }\end{array}$ & $\begin{array}{l}\text { Inten- } \\
\text { sity a }\end{array}$ \\
\hline \multicolumn{6}{|c|}{ Pentachlorobenzene } \\
\hline $\begin{array}{r}\mu \\
3.21 \\
3.25 \\
3.63 \\
3.90 \\
\text { e } 5.79 \\
6.09 \\
\text { e } 6.32 \\
\text { e } 6.42 \\
\text { e } 6.53 \\
6.94 \\
7.15 \\
7.49 \\
7.70 \\
8.08 \\
8.17 \\
8.30 \\
8.57\end{array}$ & $\begin{array}{l}c m^{-1} \\
3,115 \\
3,077 \\
2,755 \\
2,564 \\
1,727 \\
1,642 \\
1,582 \\
1,558 \\
1,531 \\
1,441 \\
1,399 \\
1,335 \\
1,299 \\
1,238 \\
1,224 \\
1,204 \\
1,167\end{array}$ & $\begin{array}{r}\% \\
45 \\
45 \\
25 \\
25 \\
45 \\
30 \\
60 \\
95 \\
95 \\
65 \\
100 \\
100 \\
75 \\
50 \\
80 \\
80 \\
100\end{array}$ & $\begin{array}{r}\mu \\
8.92 \\
9.22 \\
9.40 \\
9.52 \\
10.11 \\
10.31 \\
10.64 \\
\text { f } 11.60 \\
12.17 \\
12.54 \\
14.29 \\
14.70 \\
17.79 \\
17.92 \\
19.09 \\
19.36 \\
36.80\end{array}$ & $\begin{array}{c}\mathrm{cm}^{-1} \\
1,121 \\
1,084 \\
1,064 \\
1,050 \\
989 \\
970 \\
940 \\
862 \\
822 \\
797 \\
700 \\
680 \\
562 \\
558 \\
524 \\
516 \\
272\end{array}$ & $\begin{array}{r}\% \\
60 \\
100 \\
40 \\
40 \\
25 \\
30 \\
45 \\
100 \\
100 \\
35 \\
80 \\
100 \\
90 \\
80 \\
80 \\
25 \\
\mathrm{~d} 75\end{array}$ \\
\hline \multicolumn{6}{|c|}{ Hexachlorobenzene } \\
\hline $\begin{array}{r}7.14 \\
7.42 \\
7.71\end{array}$ & $\begin{array}{l}1,400 \\
1.347 \\
1,297\end{array}$ & $\begin{array}{l}30 \\
95 \\
65\end{array}$ & $\begin{array}{r}\text { f } 10.75 \\
13.92 \\
14.37\end{array}$ & $\begin{array}{l}930 \\
718 \\
696\end{array}$ & $\begin{array}{l}25 \\
30 \\
85\end{array}$ \\
\hline
\end{tabular}

a Intensities not corrected for background b $0.05-\mathrm{mm}$ cell.

c $0.40-\mathrm{mm}$ cell.

Solid in solution of $\mathrm{CCl}_{4}$ and band partially contributed by $\mathrm{CCl}_{4}$.

$f$ Solid in solution of $\mathrm{CS}_{2}$ and band partially contributed by $\mathrm{CS}_{2}$.

TABLE 2. Fundamental vibrations of monohalobenzenes in $\mathrm{cm}^{-1}$

\begin{tabular}{|c|c|c|c|c|c|c|c|}
\hline \multicolumn{2}{|c|}{$\mathrm{D}_{6 \mathrm{~h}}$} & $\begin{array}{c}\text { Ben- } \\
\text { zene a }\end{array}$ & $\begin{array}{l}\text { Band } \\
\text { types }\end{array}$ & $\begin{array}{l}\text { Fluoro- } \\
\text { benzene }\end{array}$ & $\begin{array}{l}\text { Chloro- } \\
\text { benzene }\end{array}$ & $\begin{array}{l}\text { Bromo- } \\
\text { benzene }\end{array}$ & $\begin{array}{c}\text { Iodo- } \\
\text { benzene }\end{array}$ \\
\hline & $A_{1 g}$ & $\begin{array}{c}3,062 \\
092\end{array}$ & $\mathrm{~A}_{1}$ & & & & 097 \\
\hline & $\begin{array}{l}A_{2 g} \\
A_{2 g}\end{array}$ & $\mathrm{~b}_{1}, 340$ & $\begin{array}{l}A_{1} \\
B_{1}\end{array}$ & $\begin{array}{l}1,019 \\
\text { (c) }\end{array}$ & 1,002 & 1,002 & (c) \\
\hline & $\mathrm{A}_{2 \mathrm{u}}$ & 671 & $\mathrm{~B}_{2}$ & & & & (c) \\
\hline & $\mathrm{B}_{1 \mathrm{u}}$ & 3,071 & $\mathbf{A}_{1}$ & &  & -... & -- \\
\hline$\nu_{6}$ & $\mathrm{~B}_{1 \mathrm{u}}$ & b1. 008 & $A_{1}$ & 807 & 701 & 668 & 653 \\
\hline & & & $\mathrm{B}_{2}$ & 759 & 740 & 735 & 729 \\
\hline$\nu_{8}$ & $\mathrm{~B}_{2 \mathrm{~K}}$ & 703 & $\mathrm{~B}_{2}$ & 684 & 682 & 681 & 681 \\
\hline $\begin{array}{l}\nu_{9} \\
\nu_{10}\end{array}$ & $\begin{array}{l}\mathrm{B}_{2 \mathrm{u}} \\
\mathrm{B}_{2 \mathrm{u}}\end{array}$ & $\begin{array}{l}\mathrm{b} 1,150 \\
\mathrm{~b} 1,310\end{array}$ & $\begin{array}{l}\mathrm{B}_{1} \\
\mathrm{~B}_{1}\end{array}$ & 1. 155 & 1,175 & 1,160 & 1,159 \\
\hline & $\mathrm{E}_{1}$ & 849 & $\mathrm{~A}_{2}$ & & & & \\
\hline & & & $\mathrm{B}_{2}$ & (c) & (c) & (c) & (c) \\
\hline$\nu_{12}$ & $\mathrm{E}_{1 \mathrm{u}}$ & 3,063 & $\begin{array}{l}A_{1} \\
B_{1}\end{array}$ & $\begin{array}{l}3,086 \\
3,068\end{array}$ & & & . \\
\hline$\nu_{13}$ & $\mathrm{E}_{1 \mathrm{u}}$ & 1,485 & $\mathrm{~A}_{1}$ & 1,495 & 1,475 & 1,475 & 1,468 \\
\hline & $F_{-}$ & 1037 & $\mathrm{~B}_{1}$ & $\begin{array}{l}1,326 \\
1,019\end{array}$ & 1,326 & $\begin{array}{l}1,324 \\
1,022\end{array}$ & $\begin{array}{l}1,321 \\
1015\end{array}$ \\
\hline$\nu_{14}$ & $L_{10}$ & 1,00 & $\begin{array}{l}A_{1} \\
B_{3}\end{array}$ & 1,019 & 1,027 & 1,022 & 1,015 \\
\hline$\nu_{15}$ & $\mathrm{E}_{2 \mathrm{~g}}$ & 3,047 & $\begin{array}{l}A_{1} \\
B_{1}\end{array}$ & $\begin{array}{l}1,220 \\
3,040\end{array}$ & $\begin{array}{l}1,085 \\
3,030\end{array}$ & $\begin{array}{l}1,071 \\
3,040\end{array}$ & $\begin{array}{l}1,060 \\
3,030\end{array}$ \\
\hline$\nu_{16}$ & $\mathrm{E}_{2 \mathrm{~g}}$ & 1,585 & $\mathrm{~A}_{1}$ & 1,592 & $1,: 80$ & $1,: 855$ & 1,577 \\
\hline & & & $\mathrm{B}_{1}$ & 1,495 & 1,475 & 1,475 & \\
\hline & W2g & 1,178 & $\begin{array}{l}A_{1} \\
B_{1}\end{array}$ & $\begin{array}{l}1,100 \\
1,064\end{array}$ & 1. 068 & $\begin{array}{l}\text { 1. } 071 \\
\text { 1. }\end{array}$ & $\begin{array}{l}1,1060 \\
1,00\end{array}$ \\
\hline$\nu_{18}$ & $\mathrm{E}_{2 \mathrm{~g}}$ & 606 & $\mathrm{~A}_{1}$ & 519 & 418 & 314 & \\
\hline & $\mathrm{E}_{2 \mathrm{u}}$ & b975 & $\begin{array}{l}\mathrm{B}_{1} \\
\mathrm{~A}_{2}\end{array}$ & 6144 & 617 & 614 & 613 \\
\hline & & & $\mathrm{B}_{2}$ & 989 & 984 & 964 & 984 \\
\hline & $\mathrm{E}_{2} \mathrm{u}_{\mathrm{u}}$ & 404 & $\begin{array}{l}\mathrm{A}_{2} \\
\mathrm{~B}_{2}\end{array}$ & $\begin{array}{l}406 \\
500\end{array}$ & $\begin{array}{l}393 \\
469\end{array}$ & 458 & 448 \\
\hline
\end{tabular}

a Benzene assignments and wave numbers from R. D. Mair and D. F. Hornig, J. Chem. Phys. 17, 1236 (1949)

Polvent.

c Band lies below the range of observations. 
TABLE 3. Assignments of some of the bands of polychlorobenzene

\begin{tabular}{|c|c|c|c|c|c|c|c|c|c|c|c|c|c|c|}
\hline \multicolumn{3}{|c|}{ Orthodichlorobenzene } & \multicolumn{3}{|c|}{ Metadichlorobenzene } & \multicolumn{3}{|c|}{ Paradichlorobenzene } & \multicolumn{3}{|c|}{ 1,2,3-Trichlorobenzene } & \multicolumn{3}{|c|}{ 1,2,4-Trichlorobenzene } \\
\hline $\begin{array}{c}\text { Wave } \\
\text { number }\end{array}$ & $\begin{array}{l}\text { Identifi- } \\
\text { cation }\end{array}$ & $\begin{array}{c}\text { Inten- } \\
\text { sity }\end{array}$ & $\begin{array}{c}\text { Wave } \\
\text { number }\end{array}$ & $\begin{array}{l}\text { Identifi- } \\
\text { cation }\end{array}$ & $\begin{array}{c}\text { Inten- } \\
\text { sity }\end{array}$ & $\begin{array}{l}\text { Wave } \\
\text { number }\end{array}$ & $\begin{array}{l}\text { Identifi- } \\
\text { cation }\end{array}$ & $\begin{array}{c}\text { Inten- } \\
\text { sity }\end{array}$ & $\begin{array}{c}\text { Wave } \\
\text { number }\end{array}$ & $\begin{array}{l}\text { Identifi- } \\
\text { cation }\end{array}$ & $\begin{array}{l}\text { Inten- } \\
\text { sity }\end{array}$ & $\begin{array}{l}\text { Wave } \\
\text { number }\end{array}$ & $\begin{array}{l}\text { Identifi- } \\
\text { cation }\end{array}$ & $\begin{array}{l}\text { Inten- } \\
\text { sity }\end{array}$ \\
\hline $\begin{array}{c}c m^{-1} \\
1,610 \\
\mathrm{a} 1,577 \\
1,484 \\
1,458 \\
1,167 \\
-\end{array}$ & $\begin{array}{ll}\mathrm{A}_{1} & \mathrm{C}-\mathrm{C} \\
\mathrm{B}_{1} & \mathrm{C}-\mathrm{C} \\
\mathrm{B}_{1} & \mathrm{C}-\mathrm{C} \\
\mathrm{A}_{1} & \mathrm{C}-\mathrm{C} \\
\mathrm{A}_{1} & \mathrm{C}-\mathrm{H}\end{array}$ & $\begin{array}{r}\% \\
90 \\
95 \\
100 \\
\text { b } 55 \\
95\end{array}$ & $\begin{array}{l}\mathrm{cm}^{-1} \\
1,631 \\
\text { a } 1,580 \\
\text { a } 1,506 \\
1,449 \\
1,164\end{array}$ & $\begin{array}{ll}\mathrm{B}_{1} & \mathrm{C}-\mathrm{C} \\
\mathrm{A}_{1} & \mathrm{C}-\mathrm{C} \\
\mathrm{B}_{1} & \mathrm{C}-\mathrm{C} \\
\mathrm{A}_{1} & \mathrm{C}-\mathrm{C} \\
\mathrm{B}_{1} & \mathrm{C}-\mathrm{H}\end{array}$ & $\begin{array}{r}\% \\
75 \\
100 \\
95 \\
95 \\
65\end{array}$ & $\begin{array}{c}c m^{-1} \\
1,645 \\
\text { a } 1,582 \\
1,451 \\
1,172 \\
1,118\end{array}$ & $\begin{array}{ll}\mathrm{A}_{\mathrm{g}} & \mathrm{C}-\mathrm{C} \\
\mathrm{B}_{1 \mathrm{~g}} & \mathrm{C}-\mathrm{C} \\
\mathrm{B}_{3 \mathrm{u}} \mathrm{C}-\mathrm{C} \\
\mathrm{A}_{\mathrm{g}} \mathrm{C}-\mathrm{H} \\
\mathrm{B}_{3 \mathrm{u}} \mathrm{C}-\mathrm{H}\end{array}$ & $\begin{array}{c}\% \\
75 \\
65 \\
90 \\
55 \\
90\end{array}$ & $\begin{array}{l}c m^{-1} \\
1,488\end{array}$ & $\mathrm{~A}_{1} \quad \mathrm{C}-\mathrm{C}$ & $\%$ & $\begin{array}{l}c m^{-1} \\
1,629 \\
1,570 \\
1,497 \\
1,462 \\
1,156\end{array}$ & $\begin{array}{ll}\mathrm{A}^{1} & \mathrm{C}-\mathrm{C} \\
\mathrm{A}^{1} & \mathrm{C}-\mathrm{C} \\
\mathrm{A}^{1} & \mathrm{C}-\mathrm{C} \\
\mathrm{A}^{1} & \mathrm{C}-\mathrm{C} \\
\mathrm{A}^{1} & \mathrm{C}-\mathrm{H}\end{array}$ & $\begin{array}{r}\% \\
80 \\
100 \\
95 \\
100 \\
100\end{array}$ \\
\hline $\begin{array}{l}1,129 \\
1,037\end{array}$ & $\begin{array}{l}\mathrm{B}_{1} \quad \mathrm{C}-\mathrm{H} \\
\mathrm{A}_{1} \mathrm{C}-\mathrm{H}\end{array}$ & $\begin{array}{l}\text { b } 55 \\
\text { b } 75\end{array}$ & $\begin{array}{r}1,081 \\
\text { a } 1,040\end{array}$ & $\begin{array}{ll}\mathrm{A}_{1} & \mathrm{C}-\mathrm{H} \\
\mathrm{B} & \mathrm{C}-\mathrm{H}\end{array}$ & $\begin{array}{r}100 \\
50\end{array}$ & & & & $\begin{array}{l}1,159 \\
1,088\end{array}$ & $\begin{array}{ll}\mathrm{B}_{1} & \mathrm{C}-\mathrm{H} \\
\mathrm{A}_{1} & \mathrm{C}-\mathrm{H}\end{array}$ & $\begin{array}{r}100 \\
70\end{array}$ & & & \\
\hline (1, 006 & $A_{1}-11$ & 10 & $\begin{array}{r}1,000 \\
969\end{array}$ & $\begin{array}{ll}\mathrm{A}_{1} & \text { ring } \\
\mathrm{B}_{2} & \mathrm{C}-\mathrm{H}\end{array}$ & $\begin{array}{l}95 \\
85\end{array}$ & 1,015 & $\mathrm{~B}_{2 \mathrm{u}} \mathrm{C}-\mathrm{H}$ & 95 & $\begin{array}{l}979 \\
964\end{array}$ & $\begin{array}{ll}\mathrm{A}_{1} & \mathrm{ring} \\
\mathrm{B}_{2} & \mathrm{C}-\mathrm{H}\end{array}$ & $\begin{array}{l}35 \\
50\end{array}$ & 1,010 & $\mathrm{~A}^{1}$ ring & 70 \\
\hline 941 & $\mathrm{~B}_{2} \mathrm{C}-\mathrm{H}$ & 100 & $\begin{array}{l}\text { a } 869 \\
\text { a } 893\end{array}$ & $\begin{array}{ll}\mathrm{B}_{2} & \mathrm{C}-\mathrm{H} \\
\mathrm{A}_{2} & \mathrm{C}-\mathrm{H}\end{array}$ & $\begin{array}{r}\text { b } 90 \\
85\end{array}$ & 935 & ${ }^{\mathrm{C}} \mathrm{A}_{\mathrm{u}} \mathrm{C}-\mathrm{H}$ & 45 & & $\ldots$ & & $\begin{array}{l}868 \\
815\end{array}$ & $\begin{array}{ll}\mathrm{A}^{11} & \mathrm{C}-\mathrm{H} \\
\mathrm{A}^{11} & \mathrm{C}-\mathrm{H}\end{array}$ & $\begin{array}{l}100 \\
100\end{array}$ \\
\hline 747 & $\mathrm{~B}_{2} \quad \mathrm{C}-\mathrm{H}$ & b 85 & $\begin{array}{l}783 \\
673\end{array}$ & $\begin{array}{ll}\mathrm{B}_{2} & \mathrm{C}-\mathrm{H} \\
\mathrm{B}_{2} & \mathrm{C}-\mathrm{C}\end{array}$ & $\begin{array}{r}\text { b } 100 \\
\text { b } 85\end{array}$ & $\begin{array}{l}819 \\
685 \\
628 \\
414\end{array}$ & $\begin{array}{ll}\mathrm{B}_{1 \mathrm{u}} & \mathrm{C}-\mathrm{H} \\
\mathrm{B}_{3 \mathrm{~g}} & \mathrm{C}-\mathrm{C}-\mathrm{C}-\mathrm{C} \\
\mathrm{B}_{1 \mathrm{~g}} & \mathrm{C}-\mathrm{C}-\mathrm{C}-\mathrm{C} \\
\mathrm{A}_{\mathrm{u}} & \mathrm{C}-\mathrm{C}\end{array}$ & $\begin{array}{r}100 \\
10 \\
10 \\
\text { d } 50\end{array}$ & $\begin{array}{l}770 \\
733\end{array}$ & $\begin{array}{ll}\mathrm{B}_{2} & \mathrm{C}-\mathrm{C} \\
\mathrm{B}_{2} & \mathrm{C}-\mathrm{C}\end{array}$ & $\begin{array}{r}100 \\
90\end{array}$ & & & \\
\hline \multicolumn{3}{|c|}{ 1,3,5-Trichlorobenzene } & \multicolumn{3}{|c|}{ 1,2,3,4-Tetrachlorobenzene } & \multicolumn{3}{|c|}{ 1,2,3,5-Tetrachlorobenzene } & \multicolumn{3}{|c|}{ 1,2,4,5-Tetrachlorobenzene } & \multicolumn{3}{|c|}{ Pentachlorobenzene } \\
\hline $\begin{array}{c}\text { Wave } \\
\text { number }\end{array}$ & $\begin{array}{l}\text { Identifi- } \\
\text { cation }\end{array}$ & $\begin{array}{c}\text { Inten- } \\
\text { sity }\end{array}$ & $\begin{array}{c}\text { Wave } \\
\text { number }\end{array}$ & $\begin{array}{l}\text { Identifi- } \\
\text { cation }\end{array}$ & $\begin{array}{c}\text { Inten- } \\
\text { sity }\end{array}$ & $\begin{array}{l}\text { Wave } \\
\text { number }\end{array}$ & $\begin{array}{l}\text { Identifi- } \\
\text { cation }\end{array}$ & $\begin{array}{c}\text { Inten- } \\
\text { sity }\end{array}$ & $\begin{array}{c}\text { Wave } \\
\text { number }\end{array}$ & $\begin{array}{l}\text { Identifi- } \\
\text { cation }\end{array}$ & $\begin{array}{c}\text { Inten- } \\
\text { sity }\end{array}$ & $\begin{array}{l}\text { Wave } \\
\text { number }\end{array}$ & $\begin{array}{l}\text { Identifi- } \\
\text { cation }\end{array}$ & $\begin{array}{l}\text { Inten- } \\
\text { sity }\end{array}$ \\
\hline $\begin{array}{l}\mathrm{cm}^{-1} \\
1,610\end{array}$ & $\mathrm{E}^{1} \quad \mathrm{C}-\mathrm{C}$ & $\begin{array}{l}\% \\
85\end{array}$ & $\begin{array}{l}c m^{-1} \\
1,620 \\
1,502\end{array}$ & $\begin{array}{l}\mathrm{C}-\mathrm{C} \\
\mathrm{C}-\mathrm{C}\end{array}$ & $\begin{array}{l}\% \\
40 \\
45\end{array}$ & $\begin{array}{l}c m^{-1} \\
1,639 \\
1,570\end{array}$ & $\begin{array}{l}\mathrm{C}-\mathrm{C} \\
\mathrm{C}-\mathrm{C}\end{array}$ & $\begin{array}{r}\% \\
60 \\
100\end{array}$ & $c m^{-1}$ & & $\%$ & $\begin{array}{l}\mathrm{cm}^{-1} \\
1,642 \\
1,558\end{array}$ & $\begin{array}{l}\mathrm{C}-\mathrm{C} \\
\mathrm{C}-\mathrm{C}\end{array}$ & $\begin{array}{l}\% \\
30 \\
90\end{array}$ \\
\hline $\begin{array}{l}1,477 \\
1,186\end{array}$ & $\begin{array}{ll}\mathrm{E}^{1} & \mathrm{C}-\mathrm{C} \\
\mathrm{E}^{1} & \mathrm{C}-\mathrm{H}\end{array}$ & $\begin{array}{l}50 \\
30\end{array}$ & 1,174 & $\mathrm{C}-\mathrm{H}$ & 100 & 1,484 & $\mathrm{C}-\mathrm{C}$ & 45 & 1,062 & $\mathrm{C}-\mathrm{H}$ & 100 & & & \\
\hline 995 & $\mathrm{~A}^{1}$ ring & 35 & & & & & & & & & & & & \\
\hline 850 & $\mathrm{~A}_{2}^{11} \quad \mathrm{C}-\mathrm{H}$ & 100 & & -....... & ...... & (n...... & ….... & 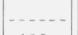 & a 877 & $\mathrm{C}-\mathrm{H}$ & 100 & a 862 & $\mathrm{C}-\mathrm{H}$ & 100 \\
\hline 662 & $\mathrm{~A}_{2}^{11} \quad \mathrm{C}-\mathrm{C}$ & 95 & 805 & $\mathrm{C}-\mathrm{H}$ & 100 & a 833 & $\mathrm{C}-\mathrm{H}$ & 100 & & & & & & \\
\hline
\end{tabular}

a Partially contributed by solvent.

b $0.05-\mathrm{mm}$ cell.

c $A_{u}$ or $B_{3 \mathrm{~g}}$.

d $1.6-\mathrm{mm}$ cell.

The most success was possible in the case of the monohalogenated benzenes. Benzene has the symmetry $\mathrm{D}_{6 \mathrm{~h}}$, and thus 20 distinct vibrational frequencies are present, 10 of which are doubly degenerate. In the monohalogenated benzenes, the symmetry is reduced to $\mathrm{C}_{2 \mathrm{v}}$. As a result, there are no degenerate frequencies remaining, and there are 36 distinct frequencies, although the $\mathrm{A}_{2}$ species will be infrared inactive. A correlation between the benzene frequencies and some of the monohalogenated benzene frequencies is given in table 2 . For fluorobenzene the published assignments ${ }^{3}$ for the gas were used as a guide.

${ }^{3}$ D. C. Smith, E. E. Ferguson, R. L. Hudson, and J. Rud Nielsen, J. Chem. Phys. 21, 1475 (1953).
For the more highly substituted chlorobenzenes more of the frequencies were inaccessible, and in each case only the $\mathrm{C}-\mathrm{C}, \mathrm{C}-\mathrm{H}$, and ring frequencies, which are more or less constant from molecule to molecule, have been designated in table 3 . These assignments should be regarded as tentative, although they were made according to the procedure suggested in the paper by Randle and Whiffen.

Washington, December 4, 1956. 